\title{
PINO The Humanoid : A Basic Architecture
}

\author{
Fuminori Yamasaki ${ }^{1,2}$, Tatsuya Matsui ${ }^{1}$ \\ , Takahiro Miyashita ${ }^{1}$, and Hiroaki Kitano ${ }^{1}$ \\ 1 Kitano Symbiotic Systems Project, ERATO, JST, \\ yamasaki@symbio.jst.go.jp, \\ ${ }^{2}$ Osaka University
}

\begin{abstract}
In this paper, we present a basic architecture and design principle behind Pino, a humanoid for RoboCup Humanoid League. Pino is designed to be a platform for research in robotics and AI. There are four major issues in Pino's design; (1) high DOF system to realize various behaviors, (2) exterior design, (3) cheap mechanical components, and (4) a basic behavioral control systems. These issues are specifically addressed in this paper in order to illustrate basic architecture and components for humanoid platform that can be widely used for many RoboCup researchers in the world.
\end{abstract}

\section{Introduction}

RoboCup Humanoid League [1], expected to start from 2002, is one of the most attractive research target, as well as potential major media attraction. We believe that the success of the humanoid league is critical for the future of RoboCup, and have major implications in robotics research and industries. Thus, we believe that it is important basic architectures for cheap and reliable research platform for humanoid to be quickly established, so that researchers in RoboCup community can initiative further investigation.

In this paper, we present an example of basic architecture of humanoid for one of RoboCup humanoid league. There are three major issues addressed in this paper.

First, humanoid should have a well designed exterior. As robot became everyday-life products, exterior designs plays major role in consumer choice. Since most existing research robots are functionally designed with minimal aesthetics, carefully designed robots are not yet exposed to general public, with exception of AIBO[2] and SIG [3]. We claim that "robot design" will be the major industrial design field, and wish to present archetype of humanoid design through Pino.

Second, the use of cheap, off-the-shelf components made humanoid more accessible for broad-range of researchers. It is a general impression that humanoid research is extremely expensive, thus it is beyond the capability of the most research group. This is partly true if we are to design all components from scratch aiming at Honda P3 [4] level humanoid. However, we believe that there is a room in the humanoid research that many interesting research can be done

P. Stone, T. Balch, and G. Kraetzschmar (Eds.): RoboCup 2000, LNAI 2019, pp. 269-278, 2001.

(C) Springer-Verlag Berlin Heidelberg 2001 
using cheap and off-the-shelf components affordable to most research groups. The hidden name of our project is, in fact, "The Poorman's humanoid project".

Third, we are working on possible modular and basic set of primitive behaviors and their control systems that are essential for most humanoids. While humanoid has very high DOF, some of primitive and basic behaviors may be definable as a hopefully modular and primitive control sub-unit. If our attempt is successful, more complex behaviors can be built on top of library of primitive high-DOF control systems.

This paper describes how we approach these issues within Pino the humanoid.

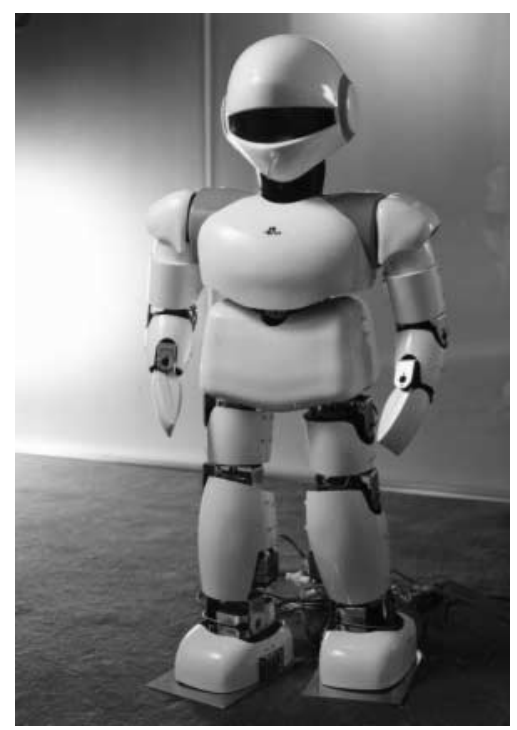

Fig. 1. PINO

\section{Exterior design}

\subsection{Necessity of Exterior Design}

Pino, the humanoid robot was developed to participation Robocup Humanoid League. The importance of exterior design is connected first and foremost to the protection of its inside system in much the same way a car or computer is similarly shielded from contact. Still, providing a mere protective shield to reduce the risk of damage to its inner systems during performance could not sufficiently express our research direction which aims to express the role of the humanoid robot in society in the future. Thus our method towards the aim of giving meaning to the existence of the robot necessitated the creation of a story to explain not only its design elements but its role in a society of the future. 


\subsection{Robot Design-"PINO" the Design Concept}

Before Pino went into development, we discussed what form, and just as importantly what size would be necessary to ensure its comfortable integration into the human home. Its size, we discovered was a very specific element of our design research which was carried out simultaneously during our primary research into its walking functions.

The scale of a fully grown adult posed a threatening presence and would, we believed cause a general sense of unease, being less a companion than a cumbersome and overpowering mechanical object. Thereupon, we judged the ideal size for such a robot would be $70[\mathrm{~cm}]$ tall; the approximate size of a one year old child taking its first steps. As for form, it was deemed necessary to design its proportions as recognizably human as possible; deviating too far from the instantly recognizable form of a human child could cause it to be seen as an altogether different object. From a psychological point of view, we noted that even the casual observer focused more attentively, and ultimately more affectionately upon a similarly structured form.

\subsection{The origin of the PINO name}

Before starting the design sketches we searched for a universal element in the representation of the human form. Images handed down from the archives of such representational forms, we believed, provided the key to integrating into the future what has universally been acknowledged as fundamental beauty. Ancient Greek sculptures and more significantly, as it turned out, the more folkloric marionettes to name a few were evoked, not only for their obvious beauty, but just as importantly for their mournful aspirations towards perfection. The marionette, with its mechanisms to facilitate movement and expression provided the ideal framework by which Pino could be adapted. Pinocchio, whose story needs no explaining, seemed an apt metaphor for our search for human qualities within the mechanical structures of our creation. We felt the necessity to place Pino somewhere in time, more specifically within the context of a story to elevate him above the static realm of the object. For this reason the infant sized cherub was discarded as a possibility despite the temptingly poetic implications of such a creature. An angel, knowing only flight finds his legs useless appendages, and Pino, whose primary function is walking, is unsuited for such dimensions. In his gestation, Pino symbolically expresses not only our desires but humankind's frail, uncertain steps towards growth and the true meaning of the word human.

\section{$3 \quad$ Structure of PINO}

\subsection{Configuration of DOFs}

Humanoid should have high DOFs to achieve various kinds of behaviors. In Robocup, for example, it search a ball and two goals, and moves to its desired location with avoiding many obstacles. Then it shoots or passes the ball. These 


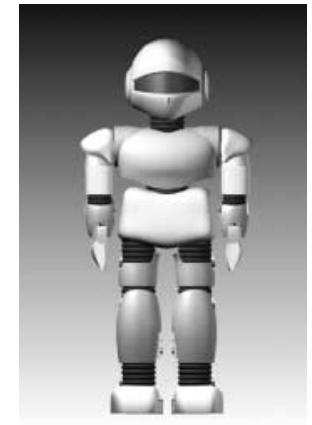

(a) Front view

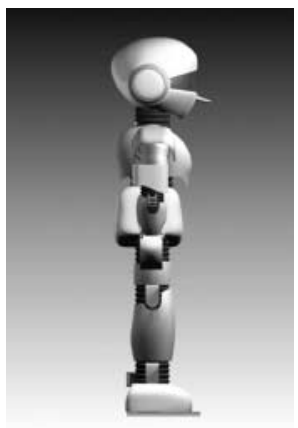

(b) Side view

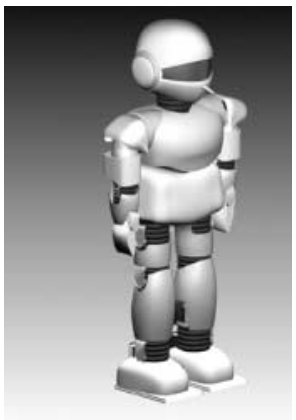

(c) Bird view

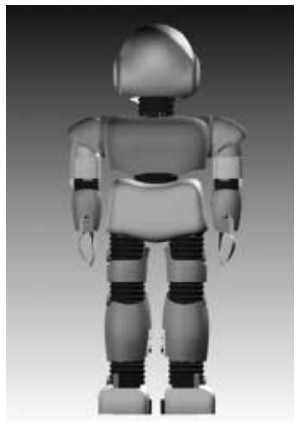

(d) Back view

Fig. 2. Computer graphic design

maneuver mainly involve following four fundamental motions; (1) keeping its body's balance, (2) moving the swing leg, (3) operating a grasping object, and (4) controlling visual attention.

To keep its body's balance, it needs 6 DOFs which denote motions of a center of gravity of itself. It also needs 6 DOFs to move the swing leg to its arbitrary location. Handling or operating a grasping object needs 6 DOFs, too. Controlling its visual attention needs 6 DOFs to move the visual sensor to arbitrary positions and orientations. Therefore humanoid needs 24 DOFs to achieve these motions in parallel. Thus, we decided the configuration of Pino as follows. Each leg has 6 DOFs, each arm has 5 DOFs, the neck has 2 DOFs and the trunk has 2 DOFs. Totally Pino has 26 DOFs (see Fig.3).

\subsection{Actuators and Sensors}

Pino has 26 motors which correspond to the number of DOFs. And it needs various kinds of sensors, For example, visual sensor for recognizing objects, posture sensor for detecting its body's balance, force and tactile sensor for detecting contact to others and falling down, and so on. If we are to design all components from scratch aiming at honda P3 level humanoid, it is extremely expensive. It is also better to use cheap components for actuators and sensors because it is considered that humanoid contact to obstacles or fall down. There are servo modules (SM) for radio control model as a cheap, compact and high torque servo motor. These SMs built in gearhead and its position controller, and these servo loops run at $50[\mathrm{~Hz}]$. These robots are already introduced which consist of SMs[5][6]. We adopted two kinds of Futaba SM for Pino which torque are $20[\mathrm{~kg} \cdot \mathrm{cm}]$ and 8 $[\mathrm{kg} \cdot \mathrm{cm}]$ (see Fig.4 (a)). All of their gears are changed to metal for reinforcement them against high torque. Pino has 8 force sensors (FSR) (see Fig.4 (b)) attached 


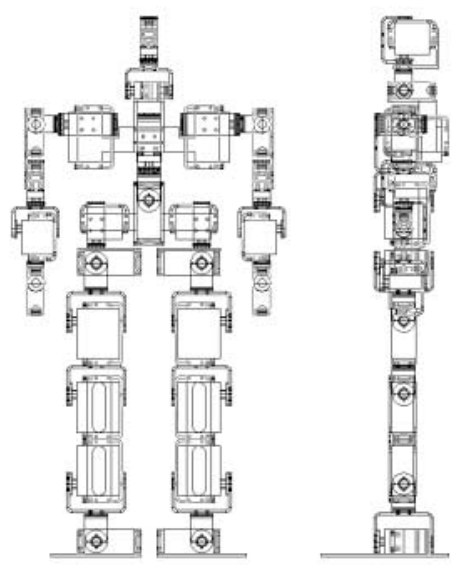

Fig. 3. Mechanical design

to each foot which can obtain foot forces, and has a posture sensor on chest so that it can sense its body's posture. we also use potentiometers in SMs as joint angle sensors. Pino also has a vision sensor mounted on head (see Fig.4 (c)). All information of these sensors are sent to host computer via A/D converters and tracking vision board.

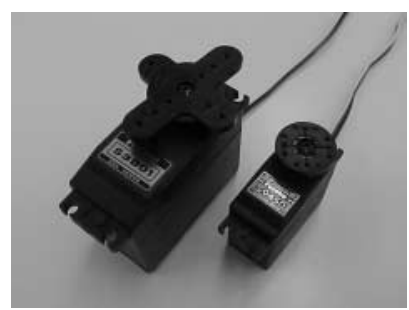

(a) Servo module

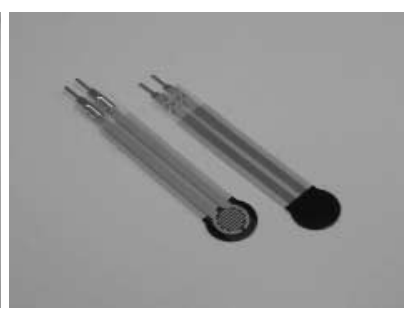

(b) Force sensor (FSR)

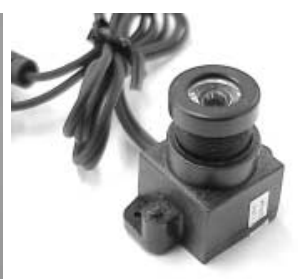

(c) Vision sensor

Fig. 4. Actuators and sensors for Pino

\subsection{Processing system}

The control system for Pino consists of a host computer and a controller of SMs. The host computer obtains all information of sensors via A/D converters and tracking vision board. The host computer consists of Pentium II 450 [MHz] processors and 512 [MByte] memory, and its operating system (OS) is realtime 
OS (RT-Linux). From these information of sensors, the host computer calculate angular velocity of each joint on realtime. Pino also has controller of itself which consists of a RISC micro-computer Hitachi SH2 (SH7050) and its slave ALTERA Programmable Logic Device (Flex10K30AQC240-3, hereafter PLD). This controller communicates the host computer via RS-232C. The PLD has 26 submodules of SM controller in it. Each submodule generates position commands for SM. The servo loops run at 50 [Hz], and the position commands for 26 SMs are updated simultaneously. We can use the development tool for this PLD for free.

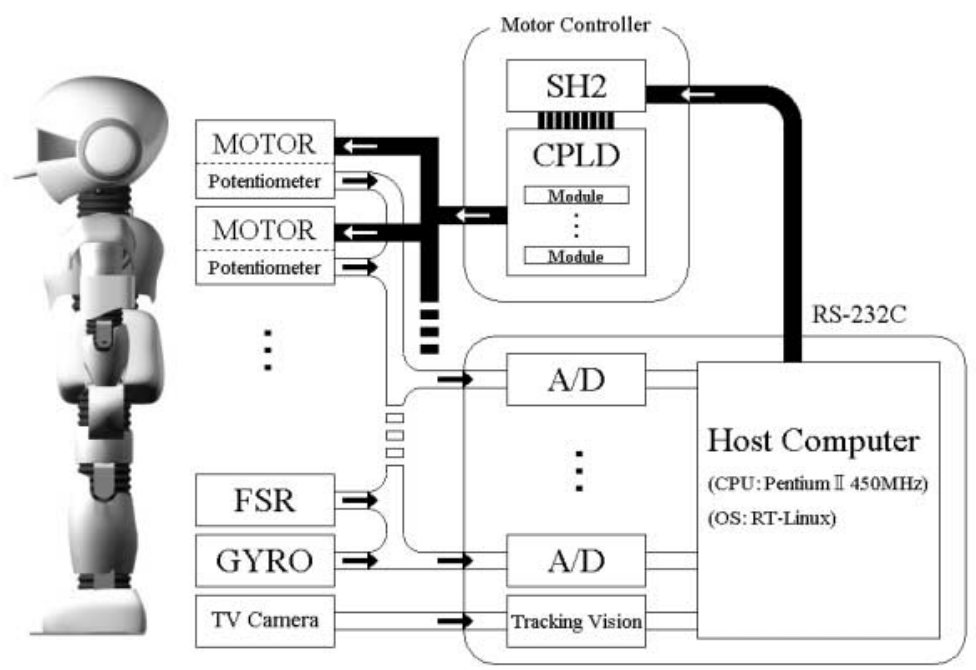

Fig. 5. System configuration

\section{Control for the PINO}

Actuators of Pino consist of 26 sets of motor and its driver for position control. However, speed control is more useful for controlling dynamic motions. Therefore, in order to realize speed control with them, we make quasi speed control system by utilizing direct kinematics of Pino and Jacobian matrices which denote velocity relationships between joint angles and positions and posture of each foot with respect to a robot coordinate frame fixed to the robot body. In this section, we explain the foot position controller of the Pino briefly.

Let $\Sigma_{R}, \Sigma_{R *}, \Sigma_{L *}, l_{R *}, l_{L *},{ }^{R} \boldsymbol{p}_{R E},{ }^{R} \boldsymbol{p}_{L E},{ }^{R} \boldsymbol{R}_{R E}$ and ${ }^{R} \boldsymbol{R}_{L E}$ denote a robot coordinate frame fixed to the robot body, link frames, link lengths of right and left legs, positions and rotation matrices of right and left legs with respect to 

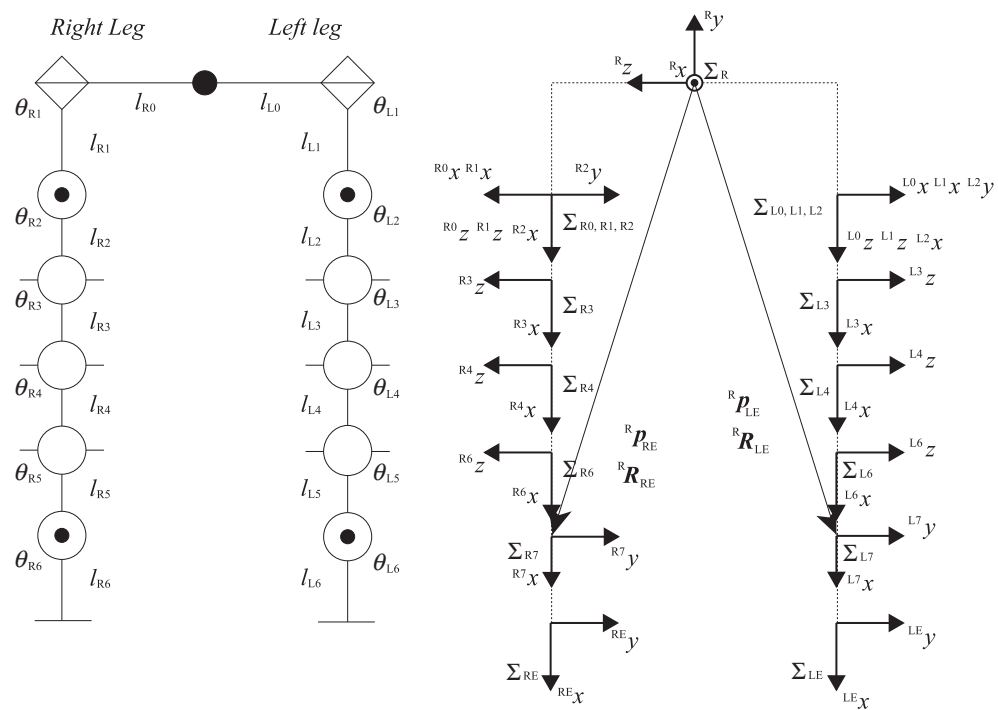

Fig. 6. Coordinate frames of legs of the Pino

the robot coordinate frame, respectively (see Fig.6). We can calculate homogeneous coordinate transformation matrices, ${ }^{R} \boldsymbol{T}_{R E}$ and ${ }^{R} \boldsymbol{T}_{L E}$ which denote the positions and posture of each foot with respect to $\Sigma_{R}$,

$$
\begin{gathered}
{ }^{R} \boldsymbol{T}_{R E}=\left[\begin{array}{cc}
{ }^{R} \boldsymbol{R}_{R E}{ }^{R} \boldsymbol{p}_{R E} \\
\boldsymbol{O} & 1
\end{array}\right]={ }^{R} \boldsymbol{T}_{R 0}{ }^{R 0} \boldsymbol{T}_{R 1}\left(\theta_{R 1}\right) \ldots{ }^{R 5} \boldsymbol{T}_{R 6}\left(\theta_{R 6}\right)^{R 6} \boldsymbol{T}_{R E}, \\
{ }^{R} \boldsymbol{T}_{L E}=\left[\begin{array}{cc}
{ }^{R} \boldsymbol{R}_{L E}{ }^{R} \boldsymbol{p}_{L E} \\
\boldsymbol{O} & 1
\end{array}\right]={ }^{R} \boldsymbol{T}_{L 0}{ }^{L 0} \boldsymbol{T}_{L 1}\left(\theta_{L 1}\right) \cdots{ }^{L 5} \boldsymbol{T}_{L 6}\left(\theta_{L 6}\right)^{L 6} \boldsymbol{T}_{L E},
\end{gathered}
$$

where ${ }^{i} \boldsymbol{T}_{j}$ denotes homogeneous coordinate transformation matrix from $\Sigma_{j}$ to $\Sigma_{i}$ calculated from link parameters. Differentiating equations (1) and (2), we

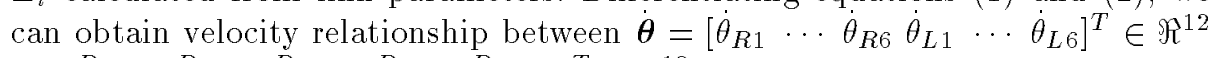
and ${ }^{R} \dot{\boldsymbol{r}}=\left[{ }^{R} \dot{\boldsymbol{p}}_{R E}{ }^{R} \boldsymbol{\omega}_{R E}{ }^{R} \dot{\boldsymbol{p}}_{L E}{ }^{R} \boldsymbol{\omega}_{L E}\right]^{T} \in \Re^{12}$,

$$
R_{\dot{r}}=\boldsymbol{J}_{r \theta}(\boldsymbol{\theta}) \dot{\boldsymbol{\theta}}
$$

where ${ }^{R} \boldsymbol{\omega}_{i}$ and $\boldsymbol{J}_{r \theta}(\boldsymbol{\theta})$ denote angular velocity vector of $\Sigma_{i}$ with respect to $\Sigma_{R}$ and $\partial^{R} \boldsymbol{r} / \partial \boldsymbol{\theta} \in \Re^{12 \times 12}$, respectively. From equation (3), a feedback controller for joint velocities to move each foot to desired positions ${ }^{R} \boldsymbol{r}_{d}$ can be derived as

$$
\boldsymbol{u}_{r}=\boldsymbol{J}_{r \theta}^{+} \boldsymbol{K}_{r}\left({ }^{R} \boldsymbol{r}_{d}-{ }^{R} \boldsymbol{r}\right)+\left(\boldsymbol{I}_{12}-\boldsymbol{J}_{r \theta}^{+} \boldsymbol{J}_{r \theta}\right) k,
$$

where $\boldsymbol{J}_{r \theta}^{+}, \boldsymbol{K}_{r}, \boldsymbol{I}_{12}$ and $k$ denote a pseudo inverse matrix of a matrix $\boldsymbol{J}_{r \theta}$, a gain matrix, a $12 \times 12$ identity matrix and an arbitrary vector that describes redundancy of the robot with respect to this servoing task. An input vector of 
the actuators of Pino is, however, a joint angular vector. Therefore we make the input vector by multiplying $\boldsymbol{u}_{r}$ by sampling rate $\triangle \boldsymbol{T}$ and adding previous joint angular vector. The block diagram of it is shown in Fig.7.

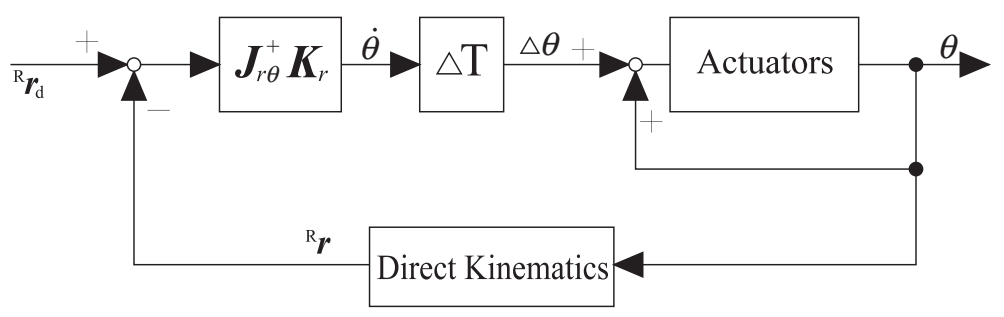

Fig. 7. A block diagram of controlling foot positions

If Pino goes into the real world and achieve their complicated tasks described in section 1, it needs capabilities to generate many motions and adapt to various situations of the environment. In previous work for the legged robot, Miyashita et al. [7] proposed a reflexive walk of a quadruped robot based on reflexes to realize an adaptive walk in a dynamic environment. They applied two reflexes, a vision-cued swaying reflection and a reflective gait, to the robot. A combination of them makes the robot walk reflexively without programming the exact motion of each joint of the legs.

\section{Experiments and Discussion}

In this section, We had the preliminary experiment that we made Pino walk to give the trajectory of each joint in order to verify the Pino's system performance. We built a kinematic model of Pino and applied it the controller of positions and posture of each foot described in section 4 . We assume that the motion sequence
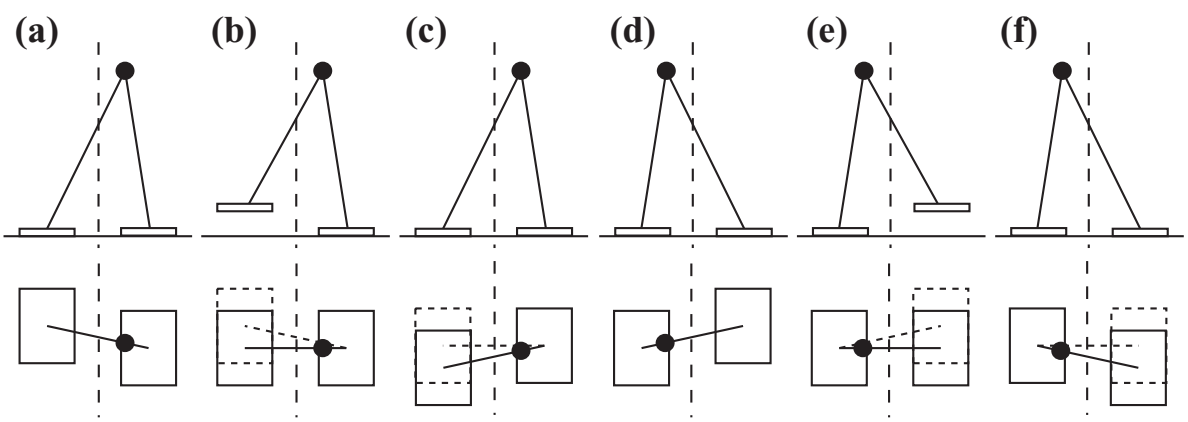

Fig. 8. The motion sequence of one cycle 


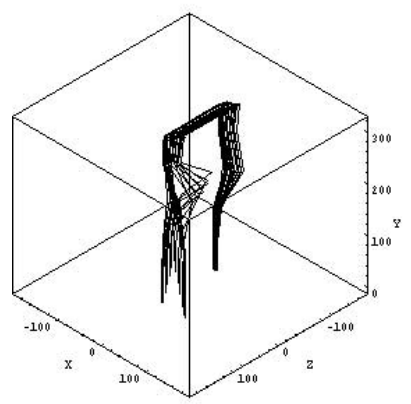

Fig. 9. Trajectories of each joint (the first step)

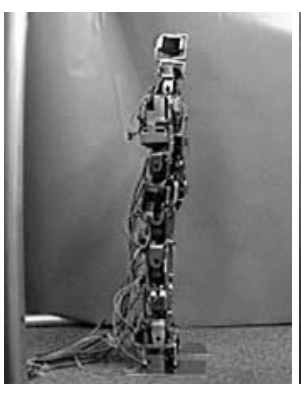

(a)

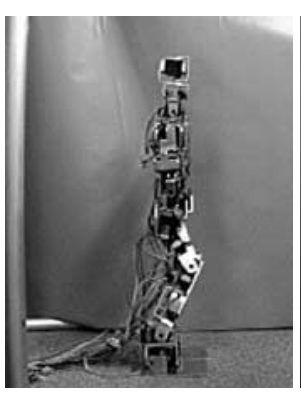

(b)

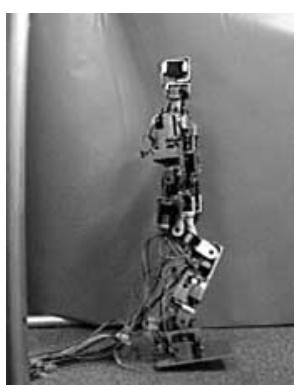

(c)

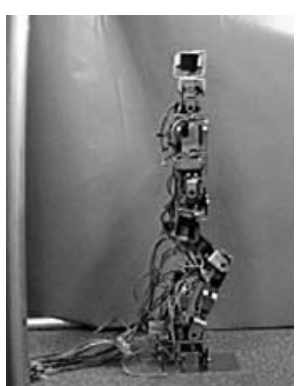

(d)

Fig. 10. Experimental result (the first step)

of one cycle of walking consists of 6 phases (see Fig.8). We generated the trajectories based on the motion sequence in advance. The example of trajectories of each joint is shown in Fig.9. We gave the trajectories to Pino, and made it walk in the real environment. As the result, Pino can walk based on them. Fig.9, Fig.10 show the first step of the result of this experiment.

In this experiment, Pino often falls down because we only use the interoceptive information. Therefore it needs to use exteroceptive information in order to realize adaptive walk. Besides, it needs to make the online system which can generate various kinds of behavior using them.

\section{Conclusion}

In this paper, we described how we approach our issues within Pino the humanoid. Pino was designed so that it is enable for most researchers to study for 
the RoboCup Humanoid League or some humanoid researches. It consists of 26 SMs, 8 force sensors, a posture sensor, a vision sensor and its control system, and all of them are cheap components. In the preliminary experiments, we verified the performance of Pino that it can walk in the real environment.

In the future work, we would like to make the online system which can generate various kinds of behavior based on exteroceptive information using a vision sensor, force sensors, a posture sensor and potentiometers.

\section{References}

1. Kitano, H., Asada, M.: RoboCup Humanoid Challenge: That's One Small Step for A Robot, One Giant Leap for Mankind Proc. of International Conference on Intelligent Robots and Systems (IROS-98) (1998)

2. Fujita, M., Kitano, H.: Development of an Autonomous Quardruped Robot for Robot Entertainment. Autonomous Robots, 5, (1998)

3. Kitano, H., Okuno, H. G., Nakadai, K., Fermin, I., Sabish, T., Nakagawa, Y., Matsui, T.: Designing a Humanoid Head for RoboCup Challenge Proc. of Agent 2000 (2000)

4. Hirose, M., Takenaka, T., Gomi, H., Ozawa, N.: Humanoid Robot. The Journal of the Robotics Society of Japan (JRSJ) vol.15, no.7 (1997) (in Japanese)

5. Inaba, M., Kanehiro, F., Kagami, S., Inoue, H.: Two-armed Bipedal Robot that can Walk, Roll Over and Stand up. Proc. of International Conference on Intelligent Robots and Systems (IROS-95) (1995)

6. Inaba, M., Igarashi, T., Kagami, S., Inoue, H.: A 35 DOF Humanoid that can Coordinate Arms and Legs in Standing up, Reaching and Grasping an Object. Proc. of International Conference on Intelligent Robots and Systems (IROS-95) (1995)

7. Miyashita, T., Hosoda, K., Asada, M.: Reflective walk based on lifted leg control and vision-cued swaying control. Proc. of 1998 BSMEE International Symposium on Climbing and Walking Robots (CLAWAR'98) (1998) 